\title{
Eigenstress model for surface stress of solids
}

\author{
Tong-Yi Zhang, * Zhi-Jia Wang, and Wing-Kin Chan \\ Department of Mechanical Engineering, Hong Kong University of Science and Technology, Clear Water Bay, Kowloon, Hong Kong, \\ China \\ (Received 28 January 2010; published 24 May 2010)
}

\begin{abstract}
Solid films are taken here as a typical example to study surface stress of solids. When a thin film is created by removing it from a bulk material, relaxation occurs inevitably because of high energy of newly created surfaces. We separate the relaxation process into normal and parallel relaxations and propose an eigenstress model to calculate the strain energy released during parallel relaxation. After parallel relaxation, a tensile (or compressive) surface eigenstress causes a compressive (or tensile) initial strain in the thin film with respect to its bulk lattice. Due to initial deformation, surface energy density and surface stress are both dependent on the film thickness, whereas surface elastic constants are independent of the film thickness. The nominal modulus of a thin film is determined by nonlinear elastic properties of its core and surfaces with initial strain. A tensile (or compressive) eigenstress makes the nominal modulus of a thin film larger (or smaller), resulting in the thinner, the harder (or softer) elastic behavior in thin films. Atomistic simulations on $\mathrm{Au}(001), \mathrm{Cu}(001), \mathrm{Si}$ (001), and diamond (001) thin films verify the developed eigenstress model.
\end{abstract}

DOI: 10.1103/PhysRevB.81.195427 PACS number(s): 46.25. $-\mathrm{y}, 46.35 .+\mathrm{z}, 73.50 .-\mathrm{h}, 68.35 . \mathrm{Md}$

\section{INTRODUCTION}

Surface stress and surface energy of solids plays a central role in the thermodynamics of solid surfaces, which offer descriptions of macroscopic phenomena without the detailed knowledge about the underlying atomistic processes. The role becomes even more significant in nanomaterials, where the surface-to-bulk ratio is much larger than that in bulk materials. Surface stress of solids has been studied by many researchers over a half century. ${ }^{1-3}$ A milestone in the study was accomplished by Shuttleworth ${ }^{1}$ who systematically analyzed surface stress and surface energy of a solid. His analysis is appropriate for bulk materials with free surfaces, where the surface transition zone is sufficiently smaller than the dimension of the underlying crystal. For an isotropic solid, Shuttleworth ${ }^{1}$ had the relationship between surface stress $\sigma^{s}$ and surface energy density $\gamma$ as

$$
\sigma^{s}=\gamma+A(\partial \gamma / \partial A),
$$

where $A$ denotes the surface area. Similarly, anisotropic surface stress tensor was given by ${ }^{4,5}$

$$
\sigma_{i j}^{s}=\gamma \delta_{i j}+\partial \gamma / \partial \varepsilon_{i j}^{s}, \quad i, j=1,2,
$$

where $\delta_{i j}$ is the Kronecker delta symbol, $\varepsilon_{i j}^{s}$ is the surface strain, and $d A=A \delta_{i j} d \varepsilon_{i j}^{s}$. Miller and Shenoy ${ }^{6}$ introduced surface elastic constants to explain size-dependent Young's modulus in nanomaterials. They expressed surface stress as

$$
\sigma_{i j}^{s}=\sigma_{i j}^{s, 0}+c_{i j k l}^{s} \varepsilon_{i j}^{s}, \quad i, j, k, l=1,2,
$$

where $c_{i j k l}^{s}$ was the surface stiffness tensor and $\sigma_{i j}^{s, 0}$ was the surface stress tensor when the material was unstrained. Equation (1) or Eq. (2) is the well-known Shuttleworth relationship between the surface stress and the surface energy. Shenoy $^{7}$ further showed that $\sigma_{i j}^{s, 0}=\left(\gamma \delta_{i j}+\partial \gamma / \partial \varepsilon_{i j}^{s}\right)_{i j}^{s}=0$. Müller and Saúl ${ }^{8}$ gave an overview about elastic effects on surface physics. Following Gibbs' approach to interfacial excess quantities, ${ }^{23}$ Müller and Saúl ${ }^{8}$ analyzed the excess of elastic energy at an interface by decomposing stress and strain tensors into perpendicular and parallel contributions, i.e.,

$$
\omega=\omega^{\|}+\omega^{\perp}=\left(\begin{array}{ccc}
\omega_{11}^{\|} & \omega_{12}^{\|} & 0 \\
\omega_{21}^{\|} & \omega_{22}^{\|} & 0 \\
0 & 0 & 0
\end{array}\right)+\left(\begin{array}{ccc}
0 & 0 & \omega_{13}^{\perp} \\
0 & 0 & \omega_{23}^{\perp} \\
\omega_{31}^{\perp} & \omega_{32}^{\perp} & \omega_{33}^{\perp}
\end{array}\right),
$$

where $\omega$ is either stress tensor or strain tensor. Based on traction continuity and nongliding at the interface, they defined interfacial stress tensor $\sigma_{i j}^{s}$ and interfacial strain tensor $\varepsilon_{i j}^{s}$ as

$$
\begin{gathered}
\sigma_{i j}^{s}=\frac{1}{S_{A B}}\left[\int_{z_{A}}^{z_{B}} \sigma_{i j}^{\|} d V-\sigma_{i j}^{\| A} V^{A}-\sigma_{i j}^{\| B} V^{B}\right], \\
\varepsilon_{i j}^{s}=\frac{1}{S_{A B}}\left[\int_{z_{A}}^{z_{B}} \varepsilon_{i j}^{\perp} d V-\varepsilon_{i j}^{\perp B} V^{B}-\varepsilon_{i j}^{\perp B} V^{B}\right],
\end{gathered}
$$

where $S_{A B}$ is the interfacial area; $V^{A}$ and $V^{A}$ are the volumes of bulk phases $A$ and $B$ extrapolated to the dividing surface; and the integration limits, $z_{A}$ and $z_{B}$, include the entire interfacial transition zone. If no stresses exist in bulk phases $A$ and B, the definition of interfacial stress tensor in Eq. (4a) is equivalent to the interfacial or surface stress tensor definition given by $\mathrm{Ibach}^{9}$ as

$$
\sigma_{i j}^{s}=\int_{\text {interface }} \sigma_{i j}^{ل} d z .
$$

Equation (4a) indicates that the surface stress tensor is an in-plane tensor only. With the definition of strains, Eq. (4b) implies that the interfacial strain tensor for a flat surface is induced by a surface displacement normal to the surface. In terms of strains, Weissmüller and Kramer ${ }^{10}$ separated surface deformation into surface-parallel deformation (the tangential strain) and surface-normal deformation (the normal strain). With the surface stress-strain relationship, as indicated in Eq. (2), surface-parallel deformation is related to the interfacial 
stress tensor given by Eq. (4a), while surface-normal deformation is similar to the interfacial strain tensor given by Eq. (4b). Weissmüller and Kramer ${ }^{10}$ proved that surface-parallel and surface-normal deformations are both state variables in the surface free energy.

Gurtin and Murdoch, ${ }^{11,12}$ Murdoch, ${ }^{13}$ and Gurtin et al. ${ }^{14}$ developed an elegant continuum mechanics framework for elastic material surfaces. The mechanical behavior of elastic surfaces is similar to that of elastic membranes. The equation describing the mechanics force balance between surface and its underlying bulk material is now called the generalized Young-Laplace equation. ${ }^{15}$ Dingreville et al. ${ }^{16}$ and Dingreville and $\mathrm{Qu}^{17}$ also systematically studied the relationship among the surface energy, surface stress, and surface elastic constants within the scheme of continuum theory of mechanics. By expending surface and bulk energy densities into Taylor's serials with respect to strain, Dingreville et al. ${ }^{16}$ demonstrated that the overall elastic behavior of structural elements (such as particles, wires, and films) is size dependent. Although such size dependency is negligible for conventional structural elements, it becomes significant when at least one of the dimensions of the element shrinks to nanometers.

When surfaces are created by cutting a crystal along a crystallographic plane, fresh surfaces without relaxation have much higher surface energy. Energy minimization relaxation of the separated free-standing crystals occurs unavoidably, in which atoms rearrange their positions locally to lower the total energy. The relaxation reduces the surface and total energies, but may lead to a change in the lattice spacing of a nanometer-sized material, which has been observed in atomistic simulations ${ }^{18,19}$ and experiments. ${ }^{20}$ The relaxationinduced strains are called initial strains. ${ }^{22}$ For instance, Liang et $a l .{ }^{19}$ found that the simulated equilibrium strains were about $-4.8 \%,-1.6 \%$, and $-1.2 \%$ for $\mathrm{Cu}$ nanowires with a $1.25 \times 1.25 \mathrm{~nm}^{2}$ square cross section and the wire length along the [001], [110], and [111] directions, respectively. As the wire cross section decreases, the Young's modulus decreases for the [001] and [111] $\mathrm{Cu}$ nanowires, while the Young's modulus increases for the $[110] \mathrm{Cu}$ nanowires. Liang et al. ${ }^{19}$ attributed the size-dependent modulus to relaxation-induced large deformation, at which nonlinear elasticity must be used to describe the stress-strain relation. Furthermore, a large initial deformation may cause phase transformation. Diao et al. ${ }^{21}$ found in atomistic simulations that when the cross-sectional area of a [100] $\mathrm{Au}$ nanowire was less than $1.83 \times 1.83 \mathrm{~nm}^{2}$, the wire underwent a phase transformation from fcc to bet, and the initial strain increased by an order of magnitude. In general, relaxation in a solid occurs inevitably when new surfaces are created.

Zhang et al. ${ }^{22}$ reanalyzed surface energy, surface stress, and surface elastic constants of a nanowire by treating a nanowire as a composite of a three-dimensional (3D) hypothetical nanowire, namely, the core, two-dimensional (2D) geometric surfaces, and one-dimensional (1D) geometric edges. When a free-standing nanowire subjected to no external loads is at equilibrium after relaxation, the core usually presents an initial deformation along the nanowire length direction with respect to the stress-free bulk counterpart. When the initial stress field in the core was known, Zhang et $a l .{ }^{22}$ determined surface stress from the force balance. However, Zhang et al. ${ }^{22}$ did not explicitly address why and how surface energy and surface stress change with the crosssectional size of a nanowire. In the present work, we develop an eigenstress model for thin films to illustrate the mechanism. With the model, we study comprehensively the effects of surfaces on surface energy density, surface stress, surface elastic constants, and nominal modulus of a crystalline thin film. Atomistic simulations are conducted to illustrate the developed concepts.

\section{THEORETICAL ANALYSIS}

When considering an interfacial transition zone separating two phases, Gibbs ${ }^{23}$ introduced the concept of a 2D dividing interface. The contribution from the interfacial transition zone to the thermodynamic properties is defined as the excess over the values that will obtain if the bulk phases retain their properties constant up to the dividing interface. When one phase is vacuum, the interface is usually called surface. In the present work, we adopt the $2 \mathrm{D}$ surface concept to study surface properties of solid thin films. It is usually taken for granted that the size of a studied solid with free surfaces is sufficiently larger than the surface transition zone, which is not true for extra-thin films and nanowires. A film has considerably large but finite in-plane dimensions along the $x$ and $y$ directions and a finite thickness along the $z$ direction. The resultant force along any virtual intersection of such a freestanding film subjected to no external loads must vanish. We may ignore the effect induced by lateral edges (surfaces) and consider only two film surfaces, each of which is associated with the surface transition zone. Then, we treat the film as a composite of a hypothetical 3D film, called the core plate or the core, and two 2D geometric surfaces. The 3D core and 2D surfaces are all assumed to be homogeneous, which is necessary for the study of the continuum concepts of surface energy, surface stress, surface elastic constants, and elastic modulus of thin films. To clearly demonstrate the physics picture, we consider square-shaped (001) films of crystals in the cubic crystal system, in which the symmetry of the crystals and the symmetry of the film orientation simplify the mathematical analysis greatly. For thin films, the plane-stress condition holds along the film thickness direction, which leads to biaxial stress fields in the (001) films. The orthogonal coordinators of the $x, y$, and $z$ axes are set, respectively, along the [100], [010], and [001] lattice directions for the (001) films. When such a square-shaped film is taken out from its stress-free bulk counterpart, the film has original dimensions of $h_{0} \times L_{0} \times L_{0}$, as shown in Fig. 1, and an unrelaxed potential energy $U_{t}^{u n r}$ and the film will relax to reach the equilibrium state. Following Weissmüller and Kramer's approach, ${ }^{10}$ we separate the relaxation process into normal and parallel relaxations, as shown in Fig. 1(a). In normal relaxation, the film is allowed to relax only along the direction perpendicular to the surfaces under a fixed dimension along the in-plane directions. The normal relaxation changes the film thickness from $h_{0}$ to $h_{0}+2 w_{0}$, meaning that each surface has a normal displacement $w_{0}$. The normal displacement could be positive or negative, reflecting the film is ex- 


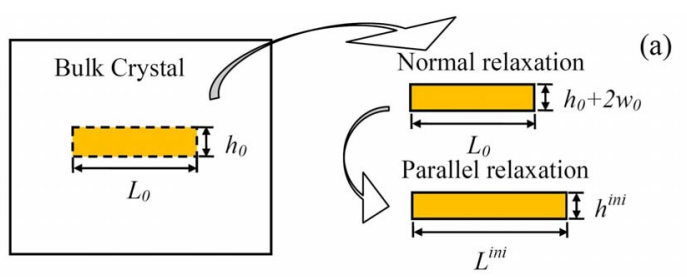

(a)

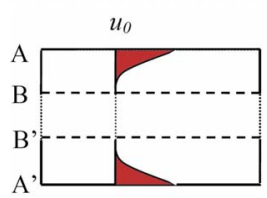

Thick film

(b)

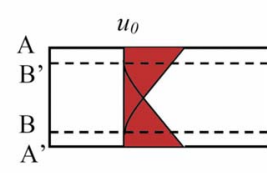

Extra thin film

(c)

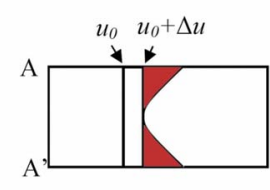

Extra thin film

(d)
FIG. 1. (Color online) (a) A film is created by removing it from a bulk material. (b)-(d) Schematic plots of energy density distributions. (b) In a thick film, the excess quantity is shown by the redshaded area, where $u_{0}$ denotes the energy density in the bulk zone, and $A B$ and $A^{\prime} B^{\prime}$ denote the surface transition zones. (c) In an extra thin film, the two surface transition zones overlap one another. The energy density of the core of a thin film is increased by $\Delta u$ compared to that in the stress-free bulk. (d) If $u_{0}+\Delta u$ is taken as a new reference state, the excess quantity shown in the shaded area will be smaller.

panded or contracted after normal relaxation. Normal relaxation reduces the potential energy of the film from $U_{t}^{u n r}$ to $U_{t}^{\perp r}=U_{t}^{u n r}-\Delta U^{\perp r}$. After normal relaxation, the in-plane constraints are released and parallel relaxation takes place with the traction-free boundary condition along the surfaces. After parallel relaxation, the film is at equilibrium with a minimum potential energy $U_{t}^{r}$ and has new dimensions of $h^{i n i} \times L^{i n i}$ $\times L^{i n i}$ with $L^{i n i}=L_{0}+\Delta L_{0}$ and $h^{i n i}=h_{0}+2 w_{0}+\Delta h_{0}$, where $\Delta h_{0}$ is due to the Poisson's ratio effect. Figures 1(b) and 1(c) illustrate the energy distribution for a thick and a thin film, respectively. When the film thickness is significantly larger than the surface transition zone size, the film interior crystal remains the same as its stress-free bulk counterpart and the excess of potential energy will be used to define the surface energy at $0 \mathrm{~K}$. For a thin film, however, the two surface transition zones overlap one another and the entire film is within the surface transition zones. In this case, how to define an excess quantity is still an open question. The relaxation-induced initial deformation gives an initial strain energy density to the core and increases the core potentialenergy density, which may be used as a new reference in the calculation of excess energy and surface energy, ${ }^{22}$ as illustrated in Fig. 1(d).

\section{A. Eigenstress model for surface stress of solids}

Similar to Dingreville et al.'s approach, ${ }^{16}$ an eigenstress model is proposed here to calculate the strain energy released during parallel relaxation, which assumes that a biaxial eigenstress $\sigma_{s}^{0}$ exists in the 2D surfaces before parallel relaxation. After parallel relaxation, an initial biaxial deformation is generated in the core plate, which gives an initial biaxial strain $\varepsilon_{c}^{i n i}=\ln \left(L^{i n i} / L_{0}\right)$. The 2D surfaces must go through the same deformation as the core does, because the surfaces coherently adhere to the core, which changes the biaxial surface stress to $\sigma_{s}^{i n i}=f_{s}^{i n i} / L^{i n i}$. The initial surface stress can be calculated by $\sigma_{s}^{i n i}=\sigma_{s}^{0}+\Delta \sigma_{s}^{i n i}$, where $\Delta \sigma_{s}^{i n i}$ denotes the parallel-relaxation-induced change in surface biaxial stress. In nonlinear elasticity, a crucial issue is the constitutive relation between stress and strain. For nonorganic crystalline materials, it is reasonable and convenient to use a quadratic constitutive relation, as used by Liang et al. ${ }^{19}$ Thus, biaxial stress in the core and the change in surface biaxial stress are calculated from $\sigma_{c}^{i n i}=Y_{c}^{*} \varepsilon_{c}^{i n i}+Y_{c}^{1^{*}}\left(\varepsilon_{c}^{i n i}\right)^{2}$ and $\Delta \sigma_{s}^{i n i}=Y_{s}^{*} \varepsilon_{c}^{i n i}$ $+Y_{s}^{1^{*}}\left(\varepsilon_{c}^{i n i}\right)^{2}$, respectively, where $Y_{c}^{*}$ and $Y_{s}^{*}$ are biaxial Young's moduli of the core and the surface, respectively, and $Y_{c}^{1^{*}}$ and $Y_{s}^{1^{*}}$ are second-order biaxial moduli of the core and the surface, respectively. Before parallel relaxation, there exists strain energy in the surfaces due to the eigenstress. After parallel relaxation, the surface stress does the work $W_{s}^{\text {in }}$ $=4 \int_{L_{0}}^{L^{i n i}} \sigma_{s} L d L$ to the surfaces and the initial strain does the work $W_{c}^{i n i}=2 \int_{L_{0}}^{L^{i n i}} \sigma_{c} L h d L$ to the core plate. Thus, parallel relaxation changes the total energy to

$$
U_{t}^{r}=U_{t}^{\perp r}+W_{c}^{i n i}+W_{s}^{i n i}
$$

At equilibrium, minimum energy requires $\partial U_{t}^{r} /\left.\partial L\right|_{L=L^{i n i}}=0$, which yields the self-balanced force equation,

$$
F^{i n i}=F_{c}^{i n i}+2 F_{s}^{i n i}=0,
$$

where $F_{c}^{i n i}=\sigma_{c}^{i n i} h^{i n i}$ and $F_{s}^{i n i}=\sigma_{s}^{i n i}=\sigma_{s}^{0}+\Delta \sigma_{s}^{i n i}$ denote core force per unit length and surface force per unit length, respectively. Equation (6a) indicates that the surface force $F_{s}^{i n i}$ acting on each of the two surfaces must be balanced by the force $F_{c}^{i n i}$ acting on the core, thereby leading to the conclusion that surface stress cannot exist alone because surface force per unit length is identical to surface stress. With the quadratic constitutive relation of stress versus strain, Eq. (6a) is rewritten as

$$
\left(h^{i n i} Y_{c}^{1^{*}}+2 Y_{s}^{1^{*}}\right)\left(\varepsilon_{c}^{i n i}\right)^{2}+\left(h^{i n i} Y_{c}^{*}+2 Y_{s}^{*}\right) \varepsilon_{c}^{i n i}+2 \sigma_{s}^{0}=0 .
$$

The solution to Eq. (6b) is given by

$$
\varepsilon_{c}^{i n i}=\frac{-\left(h^{i n i} Y_{c}^{*}+2 Y_{s}^{*}\right) \pm \sqrt{\left(h^{i n i} Y_{c}^{*}+2 Y_{s}^{*}\right)^{2}-8 \sigma_{s}^{0}\left(h^{i n i} Y_{c}^{1^{*}}+2 Y_{s}^{1^{*}}\right)}}{2\left(h^{i n i} Y_{c}^{1^{*}}+2 Y_{s}^{1^{*}}\right)} .
$$

Equation (7a) indicates that the inequality $\left(h^{i n i} Y_{c}^{*}+2 Y_{s}^{*}\right)^{2} \geq 8 \sigma_{s}^{0}\left(h^{i n i} Y_{c}^{1^{*}}+2 Y_{s}^{1^{*}}\right)$ must hold to have a real value of the initial strain. The physical argument that the initial strain must be zero if the value of eigenstress is zero makes the solution to Eq. 
(6b) have only one root, meaning that only the positive value of the square root should be taken in Eq. (7a). Then, we rewrite Eq. (7a) as

$$
\varepsilon_{c}^{i n i}=\frac{-\left(h^{i n i} Y_{c}^{*}+2 Y_{s}^{*}\right)+\left(h^{i n i} Y_{c}^{*}+2 Y_{s}^{*}\right) \sqrt{1-8 \sigma_{s}^{0}\left(h^{i n i} Y_{c}^{1^{*}}+2 Y_{s}^{1^{*}}\right) /\left(h^{i n i} Y_{c}^{*}+2 Y_{s}^{*}\right)^{2}}}{2\left(h^{i n i} Y_{c}^{1^{*}}+2 Y_{s}^{1^{*}}\right)} .
$$

The inequality $\left(h^{i n i} Y_{c}^{*}+2 Y_{s}^{*}\right)^{2} \geq 8 \sigma_{s}^{0}\left(h^{i n i} Y_{c}^{1^{*}}+2 Y_{s}^{1^{*}}\right) \quad$ means $\left|8 \sigma_{s}^{0}\left(h^{i n i} Y_{c}^{1^{*}}+2 Y_{s}^{1^{*}}\right) /\left(h^{i n i} Y_{c}^{*}+2 Y_{s}^{*}\right)^{2}\right| \leq 1$ and thus Eq. (7b) can be expressed in series of

$$
\begin{aligned}
\varepsilon_{c}^{i n i}= & \frac{-2 \sigma_{s}^{0}}{h^{i n i} Y_{c}^{*}+2 Y_{s}^{*}}-\frac{h^{i n i} Y_{c}^{1^{*}}+2 Y_{s}^{1^{*}}}{2} \frac{1}{8}\left[-\frac{8 \sigma_{s}^{0}}{\left(h^{i n i} Y_{c}^{*}+2 Y_{s}^{*}\right)^{2}}\right]^{2} \\
& +\frac{\left(h^{i n i} Y_{c}^{1^{*}}+2 Y_{s}^{1^{*}}\right)^{2}}{2} \frac{3}{46}\left[-\frac{8 \sigma_{s}^{0}}{\left(h^{i n i} Y_{c}^{*}+2 Y_{s}^{*}\right)^{2}}\right]^{3}+\cdots .
\end{aligned}
$$

Equation (7c) shows that the high-order terms in the series are induced by the nonlinear properties. We may call the term $h^{i n i} Y_{c}^{*}+2 Y_{s}^{*}$ the biaxial stiffness per unit length of the film, which is a positive parameter because strain must have the same sign as the force. Thus, the initial strain must have an opposite sign to the surface eigenstress, as shown explicitly by the first term in the right-hand side of Eq. (7c), which is the major term in the series. The result is expected because parallel relaxation is to release the surface eigenstress. The above analysis implies that the 2D surfaces might be highly strained without parallel relaxation. To reduce the high surface strain energy is the driving force for parallel relaxation. The resistance against the release of the born strain energy in the surfaces is the strain energy generated in the core plate. How much reduction in the strain energy can be achieved depends on the film thickness. For sufficiently thick films, $Y_{c}^{*} h^{i n i} \gg 2 Y_{s}^{*}$ and $Y_{c}^{*} h^{i n i}+2 Y_{s}^{*} \approx Y_{c}^{*} h^{i n i}$. With the thick film approximation, the first term in the right-hand side of Eq. (7c) shows that the initial strain in the core plate is inversely proportional to the film thickness. This means that for a sufficiently thick film, the initial strain will approach zero and there will be no parallel relaxation, as shown in Fig. 1(b). With the determined initial strain and Eq. (5), we can calculate the surface strain energy reduction caused by parallel relaxation.

Rewriting Eq. (6b) gives

$$
-F_{c}^{i n i}=2 \sigma_{s}^{0}+2 Y_{s}^{*} \varepsilon_{c}^{i n i}+2 Y_{s}^{1^{*}}\left(\varepsilon_{c}^{i n i}\right)^{2} .
$$

The core initial strain can be determined from experiments or/and atomistic calculations for thin films with different thicknesses, and the biaxial Young's modulus and the second-order biaxial Young's modulus of the bulk material should also be available from experiments or/and atomistic calculation. Thus, the force $F_{c}^{i n i}=\left[Y_{c}^{*} \varepsilon_{c}^{i n i}+Y_{c}^{1^{*}}\left(\varepsilon_{c}^{i n i}\right)^{2}\right] h^{i n i}$ acting on the core is known for each film. Then, plotting $-F_{c}^{i n i}$ versus $\varepsilon_{c}^{i n i}$ and fitting the curve with Eq. (8) determine the values of $\sigma_{s}^{0}, Y_{s}^{*}$, and $Y_{s}^{1^{*}}$.

\section{B. Surface energy}

When the stress-free state of the bulk counterpart is taken as a reference, the energy of a thin film before relaxation is given by $U_{t}^{u n r}=U_{\text {exc }}^{u n r}+U_{0}$, where $U_{0}$ and $U_{\text {exc }}^{u n r}$ denote the reference energy of the stress-free bulk and the unrelaxed excess energy, respectively. The reference energy per volume is used here and called the cohesive energy, while the reference energy per atom is also widely used in the literature. Thus, we have the surface energy density without relaxation, $\gamma^{u n r}$ $=U_{\text {exc }}^{u n r} /\left(2 L_{0}^{2}\right)$. After normal relaxation, the film energy is $U_{t}^{\perp r}$ and the excess energy and the surface energy density are reduced to $U_{e x c}^{\perp r}$ and $\gamma^{\perp r}=U_{\text {exc }}^{\perp r} /\left(2 L_{0}^{2}\right)$, respectively. Parallel relaxation further reduces the potential energy of the film from $U_{t}^{\perp r}$ to $U_{t}^{r}=U_{0}+U_{\text {exc }(s+c)}^{i n i}$, where $U_{\text {exc }(s+c)}^{i n i}$ is the total excess energy with respect to the stress-free bulk counterpart, which is distributed within the entire film because of the initial deformation of the core plate. The total excess energy may be divided into two parts: the initial core strain energy $U_{c}^{i n i}$ and the excess energy to be attributed to the surfaces, $U_{\text {exc }}^{i n i}$. Both $U_{t}^{r}$ and $U_{0}$ can be obtained from atomistic calculations, and the initial core strain energy $U_{c}^{i n i}$ can be calculated from the bulk biaxial Young's modulus, the secondorder biaxial modulus, and the initial core strain. Thus, the excess energy attributed to the surfaces is determined to be $U_{\text {exc }}^{\text {ini }}=U_{t}^{r}-U_{0}-U_{c}^{i n i}$, which yields the surface energy density of the deformed surfaces to be

$$
\gamma^{i n i}=\frac{U_{\text {exc }}^{i n i}}{2\left(L^{i n i}\right)^{2}} .
$$

Traditionally, the total excess energy is attributed to the surfaces, leading to

$$
\gamma_{(s+c)}^{i n i}=\frac{U_{e x c(s+c)}^{i n i}}{2\left(L^{i n i}\right)^{2}}
$$

The difference between Eqs. (9) and (10) may be called the pseudo-surface-energy-density,

$$
\gamma_{c}^{i n i}=\gamma_{(s+c)}^{j n i}-\gamma^{i n i}
$$

Since there is no parallel relaxation for sufficiently thick films, the pseudo-surface-energy-density will diminish as the film thickness approaches infinity. We define the parallelrelaxation-reduced surface energy density as $\Delta \gamma^{\| r}$. Then, the eigenstress model gives

$$
\gamma^{i n i}=\gamma^{\perp r}-\Delta \gamma^{\| r}-\gamma_{c}^{i n i}
$$

The parallel-relaxation-reduced surface energy density has a similar thickness-dependent behavior as the pseudo-surface- 
energy-density. Therefore, the surface energy density is a thickness-dependent property, no matter which definition of Eq. (9) or Eq. (10) is used. The thickness-dependent behavior is induced by parallel relaxation due to a nonzero eigenstress. Clearly, if eigenstress $\sigma_{s}^{0}$ is zero, no parallel relaxation will take place and the surface energy density will be $\gamma^{\perp r}$. We may further let $\gamma^{\perp r}=\gamma^{\mu n r}+\Delta \gamma^{\perp r}$ and call $\Delta \gamma^{\perp r}$ the normal-relaxation-reduced surface energy density. The present atomistic calculations show that both $\gamma^{\mu n r}$ and $\Delta \gamma^{\perp r}$ are basically independent of the film thickness.

\section{Small deformation under external loading}

For a relaxed film under external loading, the hypothetical $2 \mathrm{D}$ surfaces must undergo the same deformation as that is subjected by the core because the surfaces always adhere to the core. Applying a biaxial strain $\varepsilon^{a}$ to a thin film is equivalent to applying a biaxial force $F^{a}=2 F_{s}^{a}+F_{c}^{a}$. Unlike relaxation-induced deformation, external loading-induced deformation can be controlled. In the present study, we consider only small deformation under external loading. Under small deformation, the biaxial force is linearly proportional to the applied strain $F^{a}=\varepsilon^{a} Y_{f}^{a^{*}}$, where $Y_{f}^{a^{*}}=Y_{c}^{a^{*}} h^{i n i}+2 Y_{s}^{a^{*}}$ denotes the biaxial stiffness per unit length of the film, and $Y_{c}^{a^{*}}$ and $Y_{s}^{a^{*}}$ are the biaxial Young's moduli of the core and the surfaces, respectively, at the initial state. Since large deformation may occur during parallel relaxation, $Y_{c}^{a^{*}}$ and $Y_{s}^{a^{*}}$ depend on the initial strain of the core in such a way that

$$
\begin{aligned}
& Y_{c}^{a^{*}}=Y_{c}^{*}+2 Y_{c}^{1^{*}} \varepsilon_{c}^{i n i}, \\
& Y_{s}^{a^{*}}=Y_{s}^{*}+2 Y_{s}^{1^{*}} \varepsilon_{c}^{i n i} .
\end{aligned}
$$

With initial stresses, the surface and core stresses under a biaxial applied strain are, respectively, given by

$$
\begin{gathered}
\sigma_{s}^{a}=\sigma_{s}^{i n i}+Y_{s}^{a^{*}} \varepsilon^{a}, \\
\sigma_{c}^{a}=\sigma_{c}^{i n i}+Y_{c}^{a^{*}} \varepsilon^{a} .
\end{gathered}
$$

Equation (14a) shows how surface stress changes with an applied strain under small deformation. Comparing Eq. (14a) with Eq. (2) exhibits that both equations have the same form. If nonlinear deformation occurs during parallel relaxation, however, the surface biaxial Young's modulus depends on the initial strain, which in turn depends on the film thickness. Similar to the surface biaxial Young's modulus, the core biaxial Young's modulus is a thickness-dependent property, as shown by Eq. (13), when nonlinear parallel relaxation occurs.

The elastic work per unit surface area done by the applied strain is thus given by

$$
\underline{W}^{a}=2 \int_{L^{i n i}}^{L^{a}} F^{a} d L=Y_{f}^{a^{*}}\left(\varepsilon^{a}\right)^{2},
$$

which indicates that the elastic work is a square function of the applied biaxial strain. Thus, the biaxial stiffness per unit length of the film, $Y_{f}^{a^{*}}$, can be determined from the relation- ship between the elastic work per unit surface area and the applied strain. Once the value of $Y_{f}^{a^{*}}$ is known, we can determine the surface and bulk biaxial Young's moduli at the state of the initial strain. Furthermore, a nominal biaxial Young's modulus is defined by $Y_{n}^{a^{*}}=Y_{f}^{a^{*}} / h^{i n i}$, so that

$$
Y_{n}^{a^{*}}=Y_{c}^{a^{*}}+2 Y_{s}^{a^{*}} / h^{i n i} .
$$

Equation (16a) apparently indicates that the difference between the nominal and core biaxial Young's moduli is inversely proportional to the film thickness. If surface and core biaxial Young's moduli are both independent of the initial strain, which is true for linearly small deformation induced by parallel relaxation, Eq. (16a) gives a scaling law on how the nominal modulus changes with the film thickness. ${ }^{24}$ If nonlinear deformation occurs during parallel relaxation, however, both surface and core biaxial Young's moduli depend on the initial strain, as shown in Eq. (14). Substituting Eq. (14) into Eq. (16a) yields

$$
Y_{n}^{a^{*}}=Y_{c}^{*}+2 Y_{s}^{*} / h^{i n i}+2\left(Y_{c}^{1^{*}}+2 Y_{s}^{1^{*}} / h^{i n i}\right) \varepsilon_{c}^{i n i} .
$$

Equation (16b) shows explicitly that the nominal biaxial Young's modulus will inversely change with the film thickness only if the second-order biaxial Young's moduli vanish. In the linear case, whether a film is elastically softer or stiffer depends on the sign of the surface biaxial Young's modulus. For nonlinear elasticity, however, the nominal biaxial Young's modulus relates also to the second-order biaxial Young's moduli and the initial strain of the core as well. In the nonlinear case, the relationship between the nominal biaxial Young's modulus and the film thickness is complex because the initial strain of the core depends on the film thickness, as shown by Eq. (7b). Plugging Eq. (7b) into Eq. (16b) yields

$$
\begin{aligned}
Y_{n}^{a^{*}} & =\left(Y_{c}^{*}+\frac{2 Y_{s}^{*}}{h^{i n i}}\right) \sqrt{1+\eta} \\
& =\left(Y_{c}^{*}+\frac{2 Y_{s}^{*}}{h^{i n i}}\right)\left(1+\frac{1}{2} \eta-\frac{1}{8} \eta^{2}+\frac{3}{46} \eta^{3}+\cdots\right),
\end{aligned}
$$

where $\eta \equiv-8 \sigma_{s}^{0}\left(h^{i n i} Y_{c}^{1^{*}}+2 Y_{s}^{1^{*}}\right) /\left(h^{i n i} Y_{c}^{*}+2 Y_{s}^{*}\right)^{2}$ is a dimensionless parameter including the surface eigenstress. Equation (16c) shows explicitly that the eigenstress plays a certain role in the nominal biaxial Young's modulus if the material behavior is nonlinear. In the nonlinear case, the dimensionless parameter should be used to modify the scaling law. In this sense, we may call Eq. (16c) the general scaling law.

The elastic work per unit surface area done by an applied biaxial strain can be separated into the work done to the surfaces, $2 \underline{W}_{s}^{a}$, and the work done to the core, $\underline{W}_{c}^{a}$, so that $\underline{W}^{a}=2 \underline{W}_{s}^{a}+\underline{W}_{c}^{a}$ with $\underline{W}_{s}^{a}=\sigma_{s}^{i n i} \varepsilon^{a}+Y_{s}^{a^{*}}\left(\varepsilon^{a}\right)^{2} / 2$ and $\underline{W}_{c}^{a}=\left[\sigma_{c}^{i n i} \varepsilon^{a}\right.$ $\left.+Y_{c}^{a^{*}}\left(\varepsilon^{a}\right)^{2} / 2\right] h^{i n i}$ under small applied deformation. Thus, the surface energy density under small applied deformation as a function of an applied biaxial strain is given by 


$$
\gamma=\gamma^{i n i}+\sigma_{s}^{i n i} \varepsilon^{a}+Y_{s}^{a^{*}}\left(\varepsilon^{a}\right)^{2} / 2 .
$$

For a given film thickness, the initial surface energy density $\gamma^{i n i}$, the initial surface stress $\sigma_{s}^{i n i}$, and the initial surface modulus $Y_{s}^{a^{*}}$, have all ascertained values. However, these properties change with the film thickness, as described above. Therefore, the surface energy density under small applied deformation is size dependent for a given applied biaxial strain.

\section{Linear eigenstress model}

Under the framework of linear elasticity of core plate and surfaces, the initial biaxial stress in the core and the change in surface biaxial stress are calculated from Hooke's law and given by $\sigma_{c}^{i n i}=Y_{c}^{*} \varepsilon_{c}^{i n i}$ and $\Delta \sigma_{s}^{i n i}=Y_{s}^{*} \varepsilon_{c}^{i n i}$. The self-balanced force equation gives

$$
-Y_{c}^{*} h_{0} \varepsilon_{c}^{i n i}=2 \sigma_{s}^{0}+2 Y_{s}^{*} \varepsilon_{c}^{i n i} .
$$

Plotting $-F_{c}^{i n i}=-Y_{c}^{*} h_{0} \varepsilon_{c}^{i n i}$ versus $\varepsilon_{c}^{i n i}$ yields a slope of $2 Y_{s}^{*}$ and intersection of $2 \sigma_{s}^{0}$, meaning that the surface biaxial Young's modulus and the eigenstress are determined. Rewriting Eq. (18) gives

$$
\varepsilon_{c}^{i n i}=-\frac{2 \sigma_{s}^{0}}{Y_{c}^{*} h_{0}+2 Y_{s}^{*}} .
$$

Equation (19) can also be obtained from reduction in Eq. (7c) by letting the second-order moduli be zero. The totalenergy reduction per unit surface area in the film due to parallel relaxation is then given by

$$
\left.\underline{\Delta U^{\|}}\right|_{\min }=-\frac{2}{Y_{c}^{*} h_{0}+2 Y_{s}^{*}}\left(\sigma_{s}^{0}\right)^{2} .
$$

Equation (20) indicates that the total-energy reduction per unit surface area is linearly proportional to the square of surface eigenstress, showing again that releasing eigenstress is the driving force for parallel relaxation.

With initial stresses, the surface stress and the core stress under a biaxial applied strain are, respectively, given by

$$
\begin{aligned}
& \sigma_{s}^{a}=\sigma_{s}^{i n i}+Y_{s}^{*} \varepsilon^{a}, \\
& \sigma_{c}^{a}=\sigma_{c}^{i n i}+Y_{c}^{*} \varepsilon^{a} .
\end{aligned}
$$

Furthermore, a nominal biaxial Young's modulus is expressed as

$$
Y_{n}^{*}=Y_{c}^{*}+2 Y_{s}^{*} / h^{i n i},
$$

which shows again that whether a film is elastically softer or stiffer depends on the sign of the surface biaxial Young's modulus.

The elastic work per unit surface area done by an applied biaxial strain can be separated into the work done to the surfaces, $2 \underline{W}_{s}^{a}$, and the work done to the core, $\underline{W}_{c}^{a}$, so that $\underline{W}^{a}=2 \underline{W}_{s}^{a}+\underline{W}_{c}^{a}$ with $\underline{W}_{s}^{a}=\sigma_{s}^{i n i} \varepsilon^{a}+Y_{s}^{*}\left(\varepsilon^{a}\right)^{2} / 2$ and $\underline{W}_{c}^{a}=\left[\sigma_{c}^{i n i} \varepsilon^{a}\right.$ $\left.+Y_{c}^{*}\left(\varepsilon^{\bar{a}}\right)^{2} / 2\right] h^{\text {ini }}$. Thus, the surface energy density under an applied biaxial strain is given by

$$
\gamma=\gamma^{j n i}+\sigma_{s}^{i n i} \varepsilon^{a}+Y_{s}^{*}\left(\varepsilon^{a}\right)^{2} / 2 .
$$

The formats of Eqs. (21)-(23) are the same as those of Eqs. (14), (16a), and (17), respectively. The difference between the linear and nonlinear cases lies in the elastic constants. The initial biaxial Young's moduli are thickness dependent in the nonlinear case due to the nonlinear initial deformation, while they are correspondingly replaced with the thicknessindependent biaxial Young's moduli in the linear case.

\section{ATOMISTIC SIMULATIONS}

The goal of the present theoretical analysis is to develop a universal approach to the study of surface stress of solids within the framework of continuum concepts. Atomistic simulations are conducted to verify the developed approach. We performed atomistic simulations with LAMMPS (Ref. 25) developed at the Sandia National Laboratories.

We perform atomistic simulations for $\mathrm{Au}$ and $\mathrm{Cu}$ facecentered-cubic crystals and $\mathrm{Si}$ and diamond crystals. For $\mathrm{Au}$ and $\mathrm{Cu}$ crystals, both modified embedded-atom method (MEAM) potentials ${ }^{26,27}$ and embedded-atom method (EAM) potentials ${ }^{28,29}$ were adopted, while $\mathrm{Si}$ and diamond crystals were simulated with Tersoff potentials. ${ }^{30}$ All simulations were conducted in a molecular statics framework and implemented by using the conjugate gradient method. The orthogonal coordinators of the $x, y$, and $z$ axes are set, respectively, along the [100], [010], and [001] lattice directions for all simulations in order to be consistent with the theoretical analysis.

We first performed simulations on bulk crystals to get reference energy $U_{0}$, equilibrium lattice constant, and bulk biaxial Young's moduli. To emulate a bulk material, a representative domain of $8 \times 8 \times 8$ unit cells was adopted with periodical boundary conditions (PBCs) in all three directions. The reference energy and equilibrium lattice constant in the stress-free bulk crystals were identified through energy minimization. We simultaneously adjusted the periodic length in all three directions, i.e., changed the lattice constant along the $x, y$, and $z$ directions. At a given periodic length, we relaxed the system to reach an equilibrium state and then obtained a total potential energy. Then, the reference energy and equilibrium lattice constant were determined at the minimum of the total potential energy at equilibrium. The bulk biaxial moduli were then determined from simulations of biaxial compressive and tensile tests, which were conducted with two steps: (1) all atoms were displaced uniformly in the $x y$ plane according to the uniform biaxial strain with an increment of $0.1 \%$, and (2) the plane stress condition in the $z$ direction was identified by adjusting the periodic length along the $z$ direction to achieve the configuration with the minimum total potential energy. Subsequently, energy density versus strain was fitted by using a cubic function. Bulk biaxial Young's modulus and second-order biaxial Young's modulus were calculated from the second and third derivatives of the strain energy with respect to the applied strain. Strain ranges of $-1.0 \%$ to $1.0 \%$ for $\mathrm{Au}$ and $\mathrm{Cu}$ crystals and of $-0.5 \%$ to $0.5 \%$ for $\mathrm{Si}$ and diamond crystals were adopted in correspondence to the range of initial strains of thin films, which is discussed in the next section. 
For thin film simulations, we created a thin film by placing atoms with the stress-free bulk lattice constant. PBCs were applied only in the $x$ and $y$ directions with free surfaces in the $z$ direction to emulate a representative element of an infinitely large thin film. Film thickness $h_{0}$ was determined based on that the volume of the simulated representative film in its undistorted configuration multiplied by the density of the bulk crystal was equal to the total mass of the atoms in the thin film. ${ }^{7,31}$ The film thickness ranged from 2 to $25 \mathrm{~nm}$ and the representative element had a size of $8 \times 8$ unit cells in the $x$ and $y$ directions. The relaxation of the thin films toward the minimum-energy state was separated into two steps, i.e., normal and parallel relaxations. In normal relaxation, atoms were allowed to move in the $z$ direction to minimize the total energy with the prescribed representative film length $L_{0}$ in both $x$ and $y$ directions. After normal relaxation, parallel relaxation was conducted, in which atoms were allowed to move in all three directions. For a given number of unit cells in the representative film, the total energy of the representative film depends on the representative film length $L$. When the parallel relaxation reaches the final equilibrium state, the energy is minimized and the representative film length has its initial value $L^{i n i}$. In simulations, we changed the representative film length, i.e., changed the lattice constant along both $x$ and $y$ directions. At a given representative film length, we relaxed the film to reach an equilibrium state and obtained a total energy. Then, the initial representative film length $L^{i n i}$ was determined at the minimum of total potential energy at equilibrium. Once the initial representative film length was determined, we calculated the initial strain $\varepsilon_{c}^{i n i}$ of the thin film by using $\varepsilon_{c}^{i n i}=\ln \left(L^{i n i} / L_{0}\right)$.

In the simulations of biaxial tests on a thin film, the thin film was first relaxed to the free-standing equilibrium state. Then, an applied biaxial strain in the $x y$ plane was implemented by changing periodic length $L$ step by step from $0 \%$ to $0.3 \%$, back to $0 \%$ and to $-0.3 \%$, and then back to $0 \%$ at an increment or decrement of $0.05 \%$. After each increment or decrement, an energy minimization was conducted to ensure the simulated system to reach a new equilibrium state. The simulations show that thin films deform elastically within the applied strain range, as evidenced by the coincidence of the loading and unloading energy-strain curves and the complete recovery of the lattice structure. Subsequently, the tension stiffness of a thin film can be calculated from the second derivative of the strain energy with respect to the applied strain.

\section{RESULTS AND DISCUSSION}

\section{A. Bulk crystal properties}

Figure 2 shows the strain energy per unit volume, $\underline{U}_{c}$ $-\underline{U}_{0}$, against the applied biaxial strain $\varepsilon_{c}$ during loading and unloading under the traction-free condition along the $z$ direction, indicating that the bulk $\mathrm{Au}, \mathrm{Cu}, \mathrm{Si}$, and diamond crystals deform elastically within the applied strain range because the unloading data are completely coincided with the loading data. Furthermore, the strain energy versus the applied strain data could be perfectly fitted by a cubic function, $\underline{U}_{c}-\underline{U}_{0}=Y_{c}^{*}\left(\varepsilon_{c}\right)^{2}+\frac{2}{3} Y_{c 1}^{*}\left(\varepsilon_{c}\right)^{3}$, as shown by solid lines in Fig.
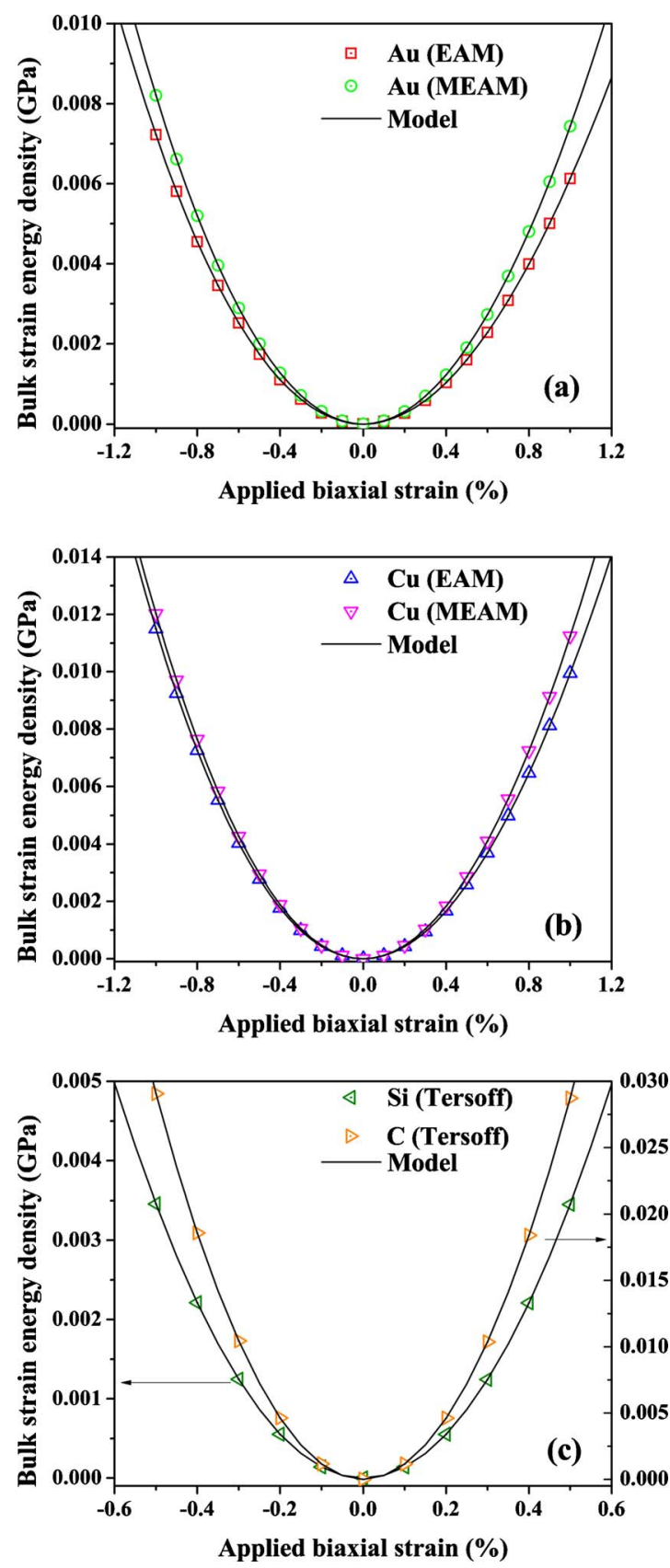

FIG. 2. (Color online) Strain energy per unit volume as a function of the biaxial strain (a) for the Au bulk crystals, (b) for the $\mathrm{Cu}$ bulk crystals, and (c) for the $\mathrm{Si}$ and diamond bulk crystals, where solid lines are fitting curves with nonlinear equation $U_{c}-U_{0}$ $=Y_{c}^{*}\left(\varepsilon_{c}\right)^{2}+\frac{2}{3} Y_{c 1}^{*}\left(\varepsilon_{c}\right)^{3}$, and biaxial strain loading is applied along the $[100]$ and [010] directions and the [001] is traction free.

2, within the investigated strain range for the $\mathrm{Au}, \mathrm{Cu}, \mathrm{Si}$, and diamond bulk crystals, where $Y_{c}^{*}$ and $Y_{c}^{1^{*}}$ are the first- and second-order biaxial Young's moduli of the bulk crystals. For the $\mathrm{Au}$ and $\mathrm{Cu}$ bulk crystals, the deformation behavior under compression is obviously different from that under tension, as shown in Figs. 2(a) and 2(b). Elastic hardening occurs under compression for simulations using both EAM and MEAM potentials. Similar nonlinear elastic deformation behaviors under compression were observed in the molecular 
TABLE I. Cohesive energy $\underline{U}_{0}$, equilibrium lattice constant $a_{0}$, and biaxial moduli $Y_{c}^{*}$ and $Y_{c 1}^{*}$ of bulk crystals.

\begin{tabular}{lcccr}
\hline \hline Bulk crystal & $\begin{array}{c}\underline{U}_{0} \\
(\mathrm{GPa})\end{array}$ & $\begin{array}{c}a_{0} \\
(\AA)\end{array}$ & $\begin{array}{c}Y_{c}^{*} \\
(\mathrm{GPa})\end{array}$ & \multicolumn{1}{c}{$\begin{array}{c}Y_{c 1}^{*} \\
(\mathrm{GPa})\end{array}$} \\
\hline $\mathrm{Au}(\mathrm{EAM})$ & -37.08 & 4.080 & 66.75 & -822.95 \\
$\mathrm{Au}(\mathrm{MEAM})$ & -37.35 & 4.070 & 78.22 & -579.36 \\
$\mathrm{Cu}(\mathrm{EAM})$ & -48.02 & 3.615 & 107.16 & -1162.22 \\
$\mathrm{Cu}(\mathrm{MEAM})$ & -47.82 & 3.620 & 116.30 & -585.16 \\
$\mathrm{Si}$ (Tersoff) & -37.02 & 5.432 & 138.16 & -33.38 \\
Diamond (Tersoff) & -208.29 & 3.566 & 1156.02 & -2074.52 \\
\hline \hline
\end{tabular}

static simulations of $\mathrm{Cu}$ bulk crystals by Liang et al. ${ }^{19}$ Then, we took the second and third derivative coefficients of the fitting results to calculate the biaxial Young's moduli of the bulk crystals and tabulated them in Table I. In addition, the calculated cohesive energy and equilibrium lattice constants of the investigated crystals are also shown in Table I. The bulk biaxial Young's moduli determined from the atomistic simulations will be utilized in the following analysis.

\section{B. Normal relaxation}

Table II lists the change in atomic layer spacing after normal relaxation. As expected, the normal-relaxationinduced contraction in the film thickness, as indicated by the normal displacement $w_{0}$, occurs only within less than four surface-atomic layers.

\section{Surface eigenstress and surface biaxial Young's moduli}

After parallel relaxation, an initial core biaxial strain $\varepsilon_{c}^{i n i}$ is generated in each of the thin films. Figure 3 illustrates core initial strains in the $\mathrm{Au}, \mathrm{Cu}, \mathrm{Si}$, and diamond films. The $\mathrm{Au}$ and $\mathrm{Cu}$ films exhibit compressive core initial strains, while tensile core initial strains appear in the Si and diamond films. The absolute values of the initial core strains increase as the films get thinner. This is because the film elastic stiffness is smaller for a thinner film and thus the resistance against the relief of the surface eigenstress is lower. The EAM potentials produce larger absolute values of core initial strains than those produced by the MEAM potentials. Moreover, the ab-

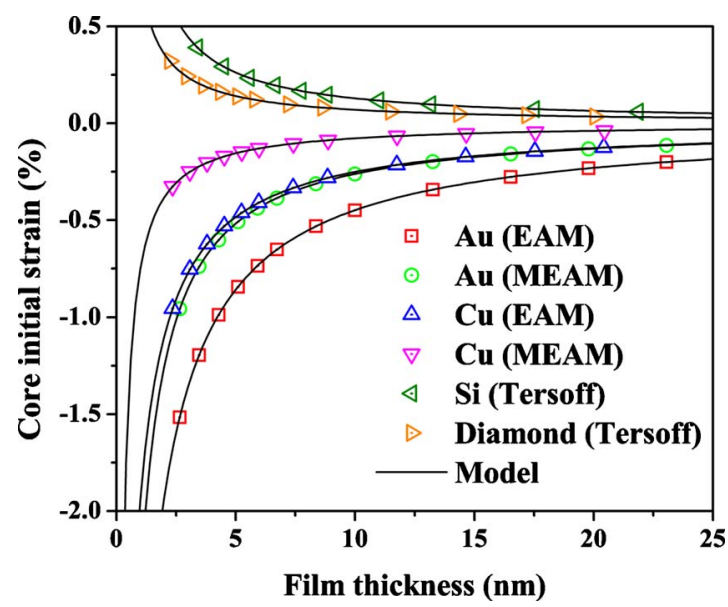

FIG. 3. (Color online) The core initial strain as a function of the film thickness, where solid lines are fitting curves with Eq. (7).

solute values of initial strains are smaller for the $\mathrm{Si}$ and diamond thin films than those of $\mathrm{Au}$ and $\mathrm{Cu}$ thin films with the same number of lattice cells in the $z$ direction. The solid lines in Fig. 3 show the prediction of the initial strain from Eq. (7b) with the values of surface Young's moduli and eigenstress determined from fitting the simulation data with Eq. (8), which is described below.

When the initial strains were known, we calculated the initial core stresses in the hypothetical thin film with the bulk modulus listed in Table I. The nonlinear eigenstress model gives the initial stress in the core plate to be $\sigma_{c}^{i n i}=Y_{c}^{*} \varepsilon_{c}^{i n i}$ $+Y_{c 1}^{*}\left(\varepsilon_{c}^{i n i}\right)^{2}$, while the initial core stress is $\sigma_{c}^{i n i}=Y_{c}^{*} \varepsilon_{c}^{i n i}$ in the linear eigenstress model. Then, we calculated the initial core force $F_{c}^{i n i}=\sigma_{c}^{i n i} h^{i n i}$ in the hypothetical thin film. Figure 4 shows the negative initial core force as a function of the core initial strain for both linear and nonlinear eigenstrain models, where the dashed and solid lines are fitting results based on Eqs. (18) and (8), respectively. Although the value of initial strain is atomic potential dependent, the simulation and fitting results demonstrate that the eigenstress model works for the three kinds of potentials. For the $\mathrm{Au}$ and $\mathrm{Cu}$ films, the results from the EAM and MEAM potentials show almost the same characteristics, except that the MEAM gives a smaller absolute value of initial core force. The value of first-order modulus of $\mathrm{Au}$ or $\mathrm{Cu}$ (100) surface depends highly

TABLE II. Normal-relaxation-induced displacement $w_{0}$ and atomic layer spacing change $\Delta z_{i, i+1}$ of the films after normal relaxation, where $i$ denotes the sequence number of an atomic layer counted from the surface.

\begin{tabular}{lcrrrrr}
\hline \hline & & \multicolumn{5}{c}{ Layer spacing relaxation $(\AA)$} \\
\cline { 3 - 7 } Film & $w_{0}(\AA)$ & \multicolumn{1}{c}{$\Delta z_{1,2}$} & \multicolumn{1}{c}{$\Delta z_{2,3}$} & \multicolumn{1}{c}{$\Delta z_{3,4}$} & $\Delta z_{4,5}$ & $\Delta z_{5,6}$ \\
\hline $\mathrm{Au}($ EAM) & -0.117 & -0.129 & 0.012 & 0.000 & 0.000 & 0.000 \\
$\mathrm{Au}(\mathrm{MEAM})$ & -0.098 & -0.117 & 0.024 & -0.005 & 0.000 & 0.000 \\
$\mathrm{Cu}(\mathrm{EAM})$ & -0.030 & -0.024 & -0.006 & 0.000 & 0.000 & 0.000 \\
$\mathrm{Cu}(\mathrm{MEAM})$ & -0.015 & -0.015 & 0.000 & 0.000 & 0.000 & 0.000 \\
$\mathrm{Si}$ (Tersoff) & -0.085 & -0.091 & 0.006 & 0.000 & 0.000 & 0.000 \\
Diamond (Tersoff) & -0.108 & -0.125 & 0.017 & 0.002 & 0.000 & 0.000 \\
\hline \hline
\end{tabular}



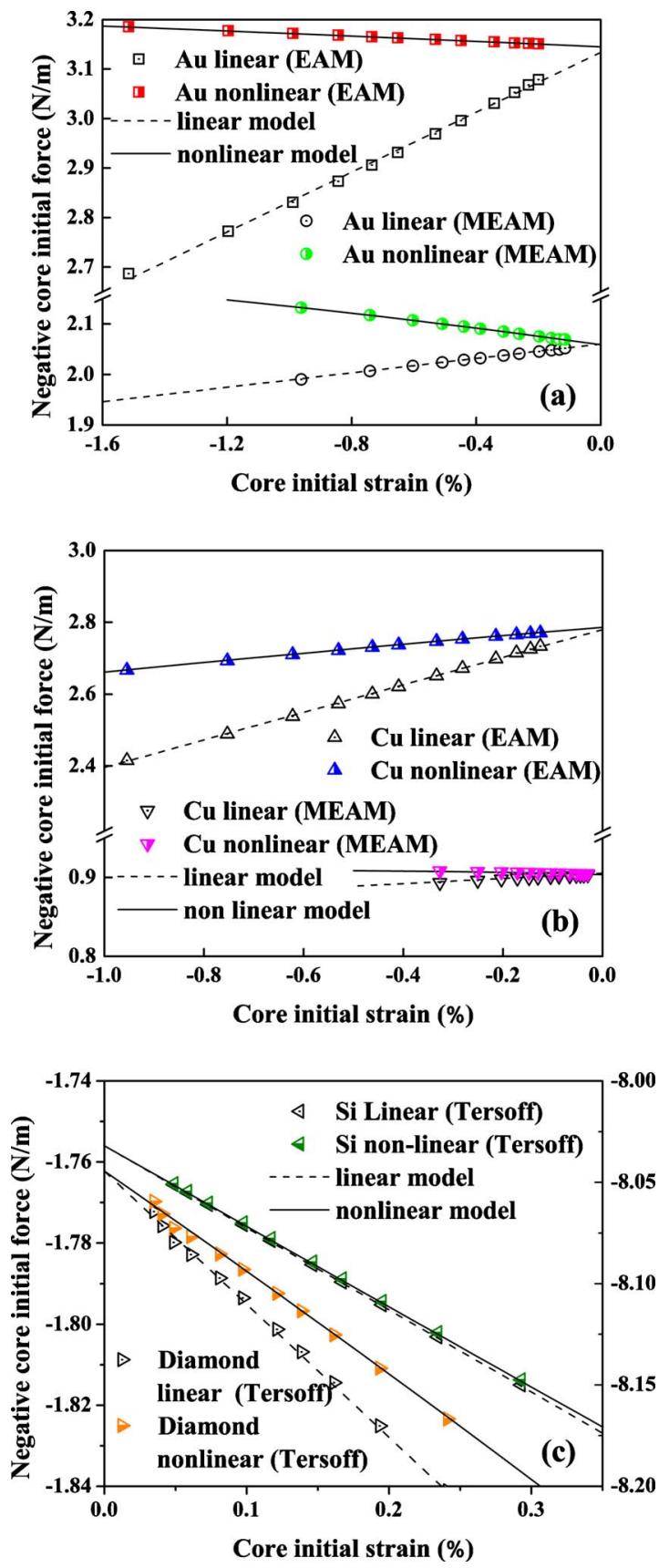

FIG. 4. (Color online) Negative core initial force versus initial strain, (a) for the $\mathrm{Au}(001)$ thin films, (b) for the $\mathrm{Cu}$ (001) thin films, and (c) for Si (001) and diamond (001) thin films, where dashed and solid lines are fitting curves with Eqs. (8) and (18) based on the nonlinear and linear eigenstress models, respectively.

on the model adopted. If the linear model is adopted, the core and the surface are both assumed to deform linearly. The slope of negative initial core force versus initial strain takes a positive value, meaning that the first-order surface Young's modulus is positive, as listed in Table III. On the other hand, if the nonlinear model is adopted, the slope will take a negative value, thereby leading to a negative first-order surface Young's modulus, as listed in Table III for the Au (EAM), Au (MEAM), and $\mathrm{Cu}$ (MEAM) films. This phenomenon can be explained from the nonlinear nature of counterpart bulk elas- ticity determined from the simulations with the EAM and MEAM potentials. Take Au crystals as an example. The second-order biaxial Young's modulus of Au crystals is about one order in magnitude larger than the first-order Young's modulus, as shown in Table I. This means that the stress will be dominated by the second-order biaxial Young's modulus at large strains. For the $\mathrm{Si}$ and diamond crystals with Tersoff potential, nonlinear terms play a minor role, as indicated by values of $Y_{c}^{*}$ and $Y_{c 1}^{*}$ in Table I and shown in Fig. 4(c). Depending on the model used, the negative initial core force of films as a function of the core initial strain curve could be fitted by a linear function of $-Y_{c}^{*} h_{0} \varepsilon_{c}^{i n i}=2 \sigma_{s}^{0}+2 Y_{s}^{*} \varepsilon_{c}^{i n i}$ for the linear eigenstress model or a quadratic function of $-F_{c}^{\text {ini }}$ $=2 \sigma_{s}^{0}+2 Y_{s}^{*} \varepsilon_{c}^{i n i}+2 Y_{s}^{1^{*}}\left(\varepsilon_{c}^{i n i}\right)^{2}$ for the nonlinear eigenstress model, which are correspondingly shown by the dashed or solid lines in Fig. 4. All determined values of $Y_{s}^{*}, Y_{s 1}^{*}$, and $\sigma_{s}^{0}$ are tabulated in Table III. For the linear model, we may define a surface biaxial eigenstrain as $\varepsilon_{s}^{0}=\sigma_{s}^{0} / Y_{s}^{*}$, which is also shown in Table III. Due to the difference in the used fitting equation between the linear and nonlinear models, the determined surface Young's moduli change greatly if nonlinear initial deformation occurs and the results are fitted by linear eigenstress model. For example, the first-order surface moduli of the Au (EAM), Au (MEAM), and Cu (MEAM) films change their sign from negative to positive when fitting equation of a quadratic function of $-F_{c}^{i n i}=2 \sigma_{s}^{0}+2 Y_{s}^{*} \varepsilon_{c}^{i n i}$ $+2 Y_{s}^{1^{*}}\left(\varepsilon_{c}^{i n i}\right)^{2}$ for the nonlinear eigenstress model is changed to a linear function of $-Y_{c}^{*} h_{0} \varepsilon_{c}^{i n i}=2 \sigma_{s}^{0}+2 Y_{s}^{*} \varepsilon_{c}^{i n i}$ for the linear eigenstress model.

\section{Surface energy density}

Just after the creation of a thin film, we calculated the unrelaxed total potential energy as $U_{t}^{u n r}$. Then, the surface energy density without relaxation is calculated from the difference between $U_{t}^{u n r}$ and the reference energy as $\gamma^{\text {unr }}$ $=\left(U_{t}^{u n r}-U_{0}\right) /\left(2 L_{0}^{2}\right)$. After normal relaxation, the total potential energy of the thin film is decreased to $U_{t}^{\perp r}$ and the surface energy density is reduced to $\gamma^{\perp r}=\left(U_{t}^{\perp r}-U_{0}\right) /\left(2 L_{0}^{2}\right)$. After parallel relaxation, the total potential energy of the thin film is further decreased to $U_{t}^{r}$ and the corresponding surface energy density $\gamma_{(s+c)}^{\text {ini }}$ is given by $\left(U_{t}^{r}-U_{0}\right) /\left(2 L_{0}^{2}\right)$. Moreover, we calculated the initial strain energy of the hypothetical thin film core from $U_{c}^{i n i}=V\left[Y_{c}^{*}\left(\varepsilon_{c}^{i n i}\right)^{2}+\frac{2}{3} Y_{c 1}^{*}\left(\varepsilon_{c}^{i n i}\right)^{3}\right]$, where $V$ is the volume of the representative film. With respect to the deformed core, the surface energy density after parallel relaxation is expressed by $\gamma^{i n i}=\left(U_{t}^{r}-U_{c}^{i n i}-U_{0}\right) /\left(2 L_{0}^{2}\right)$. The surface energy densities, $\gamma^{\mu n r}$ before relaxation, $\gamma^{\perp r}$ after normal relaxation, and $\gamma_{(s+c)}^{\text {ini }}$ and $\gamma^{i n i}$ after parallel relaxation are all plotted in Fig. 5 as functions of the film thickness. The unrelaxed surface has the highest surface energy density that is independent of the film thickness. Normal relaxation reduces the surface energy density and the reduction is also independent of the film thickness. This is because normal relaxation occurs only within few atomic surface layers, which are within a half thickness of the simulated thin films, as described in Sec. IV B. Parallel relaxation, however, occurs over the entire thin film. Although parallel relaxation is induced by redistribution of charges, it can be well described 
TABLE III. Eigenstress $\sigma_{s}^{0}(\mathrm{~N} / \mathrm{m})$, eigenstrain $\varepsilon_{s}^{0}(\%)$, surface biaxial moduli $Y_{s}^{*}(\mathrm{~N} / \mathrm{m})$, and $Y_{s 1}^{*}(\mathrm{~N} / \mathrm{m})$ of the (001) thin films determined from self-force balance.

\begin{tabular}{|c|c|c|c|c|c|c|}
\hline \multirow[b]{2}{*}{ Film } & \multicolumn{3}{|c|}{ Linear model } & \multicolumn{3}{|c|}{ Nonlinear model } \\
\hline & $\varepsilon_{s}^{0}$ & $Y_{s}^{*}$ & $\sigma_{s}^{0}$ & $Y_{s 1}^{*}$ & $Y_{s}^{*}$ & $\sigma_{s}^{0}$ \\
\hline $\mathrm{Au}(\mathrm{EAM})$ & 10.354 & 15.133 & 1.567 & -5.446 & -1.403 & 1.573 \\
\hline $\mathrm{Au}(\mathrm{MEAM})$ & 29.258 & 3.518 & 1.029 & -72.181 & -4.565 & 1.029 \\
\hline $\mathrm{Cu}(\mathrm{EAM})$ & 7.238 & 19.202 & 1.390 & -56.236 & 5.661 & 1.393 \\
\hline $\mathrm{Cu}$ (MEAM) & 27.915 & 1.621 & 0.453 & -74.687 & -0.873 & 0.452 \\
\hline Si (Tersoff) & 8.669 & -10.128 & -0.878 & 14.352 & -9.966 & -0.878 \\
\hline Diamond (Tersoff) & 12.251 & -32.832 & -4.022 & -329.175 & -24.368 & -4.022 \\
\hline
\end{tabular}

by the eigenstress model, meaning that the surface energy densities, $\gamma_{(s+c)}^{i n i}$ and $\gamma^{i n i}$, can be predicted from the eigenstress model when the surface energy density after normal relaxation, $\gamma^{\perp r}$, is known. The symbols and curves in Fig. 5 are the simulation results and theoretical predictions from the nonlinear eigenstress model, respectively, and both agree perfectly with each other. The parallel-relaxation-induced reduction in surface energy depends on the film thickness. The thinner the film is, the greater the reduction will be. That is why the surface energy density is thickness dependent. For sufficiently thick films, the surface energy densities for the simulated crystal surfaces obtained in the present work are
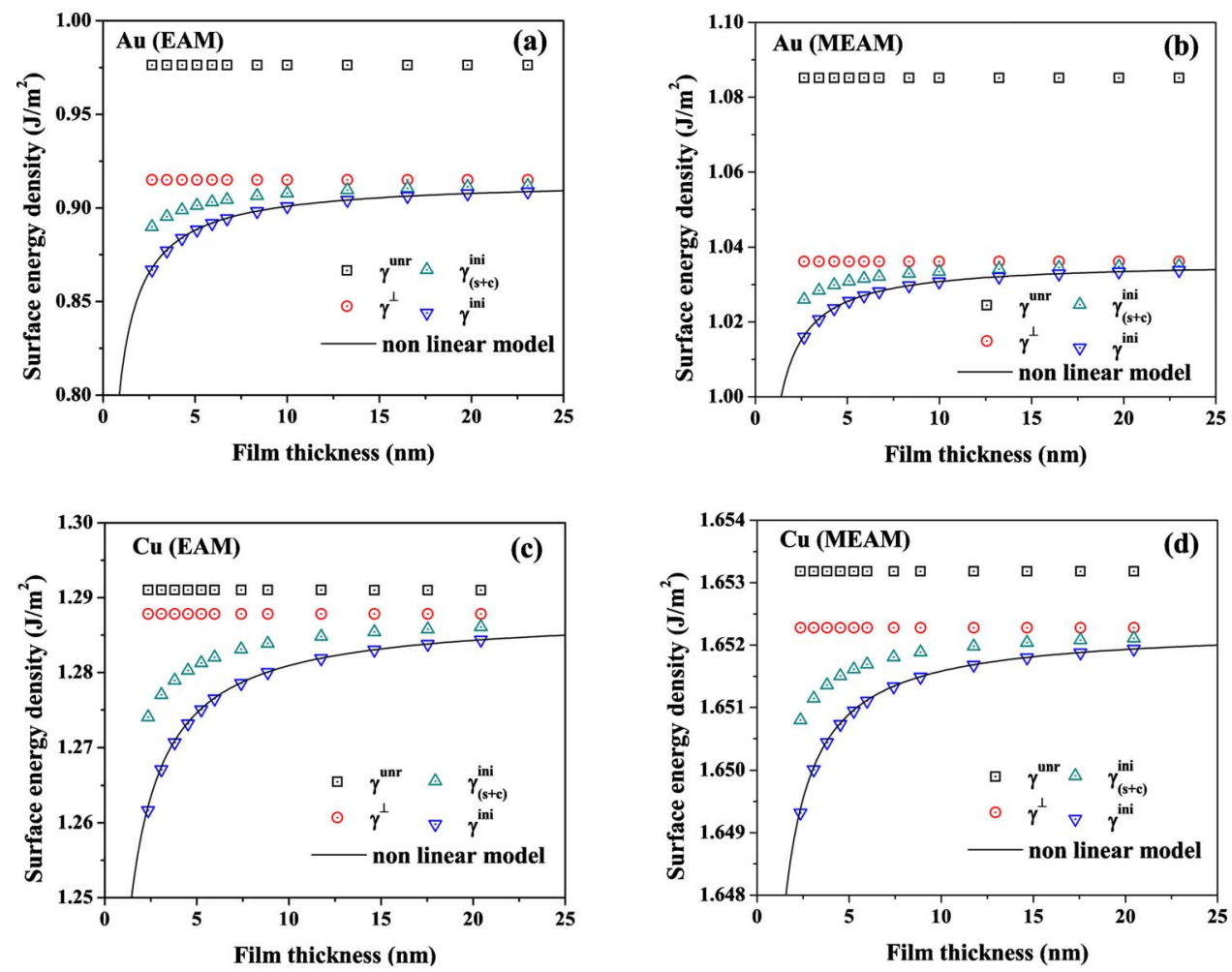

FIG. 5. (Color online) Surface energy densities in free-standing films as a function of the film thickness, where solid curves denote predictions from Eq. (12) based on the nonlinear eigenstress model.
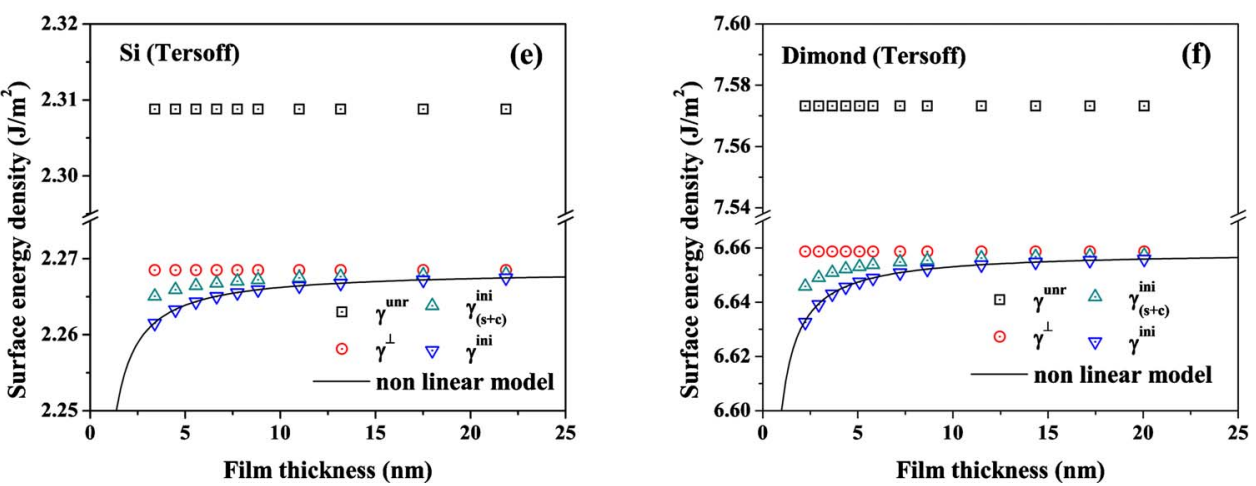

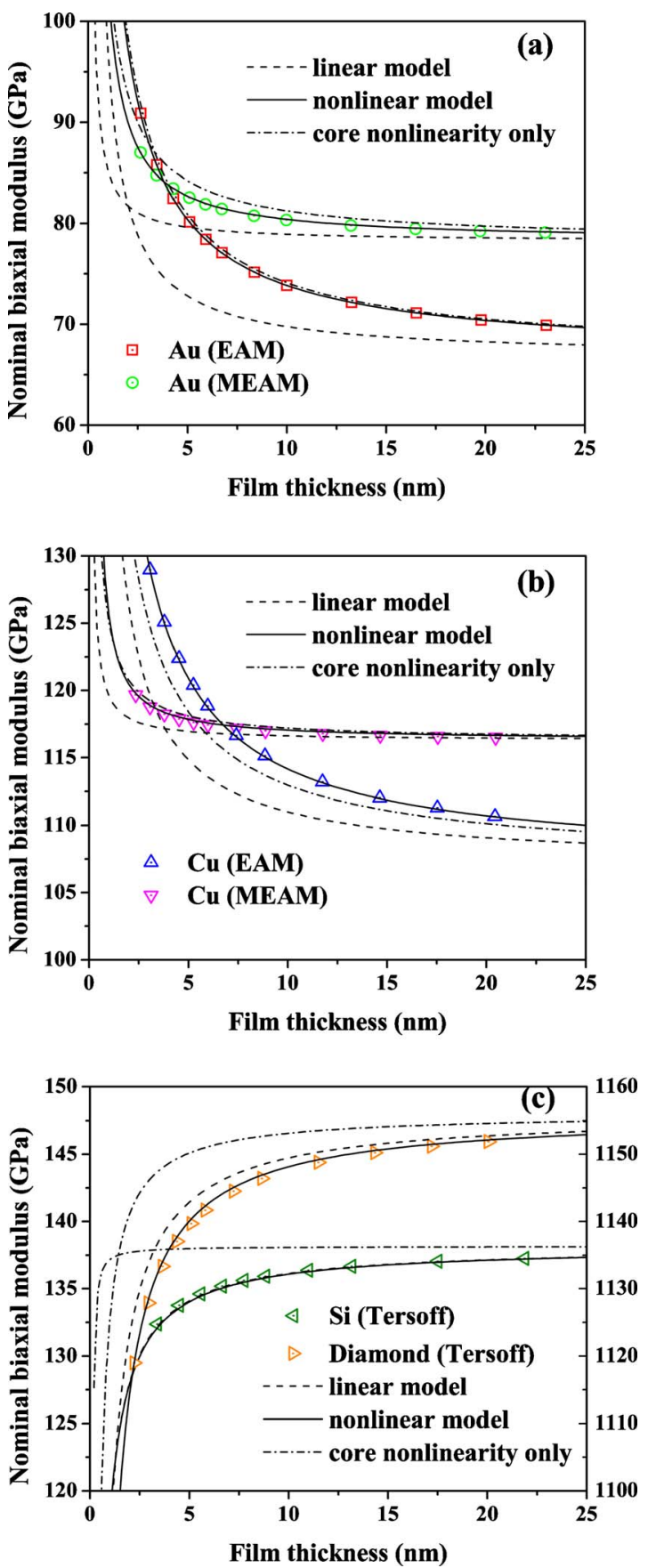

FIG. 6. (Color online) The nominal biaxial Young's modulus as a function of the film thickness, (a) for the Au (001) thin films, (b) for the $\mathrm{Cu}(001)$ thin films, and (c) for the $\mathrm{Si}$ and diamond (001) thin films, where curves are predictions from Eq. (16b) for the nonlinear eigenstress model, Eq. (22) for the linear eigenstress model, and Eq. (24) the only core-nonlinear model.

more or less the same as those reported in the literature. $^{21,29,32}$

\section{E. Size-dependent biaxial Young's modulus}

Figure 6 shows the nominal biaxial Young's modulus ver- sus the film thickness. The $\mathrm{Au}(001)$ and $\mathrm{Cu}$ (001) films illustrate the thinner the harder behavior, as shown in Figs. 6(a) and 6(b). By contrast, Fig. 6(c) shows the thinner the softer behavior of the $\mathrm{Si}(001)$ and diamond (001) films. In Fig. 6, we also plot the predictions from the nonlinear eigenstress model, the linear eigenstress model, and the only corenonlinear model used by Liang et al., ${ }^{19}$ which gives the nominal biaxial Young's modulus,

$$
Y_{n}^{a^{*}}=Y_{c}^{*}+2 Y_{c}^{1^{*}} \varepsilon_{c}^{i n i} .
$$

Figure 6 illustrates that the predictions from the nonlinear eigenstress model, with the parameters listed in Tables I and III, agree with the simulated results. Since the initial deformation in the $\mathrm{Si}$ and diamond crystals can be approximately treated to be linear, the thickness-dependent nominal biaxial Young's modulus can be described by the linear eigenstress model with a reasonable accuracy, as illustrated in Fig. 6(c). If the initial deformation in bulk crystals exhibits significant nonlinear features, the only core-nonlinear model will have the capability to catch, in a large extent, the thicknessdependent behavior of nominal Young's modulus, which is clearly shown in Figs. 6(a) and 6(b) for the $\mathrm{Au}(001)$ and $\mathrm{Cu}$ (001) films, respectively. Furthermore, if nominal Young's modulus is determined from a bending test, the thicknessdependent behavior will be much more significant, ${ }^{33,34}$ which is caused by surface elastic constants and can be explained by the eigenstress model.

\section{CONCLUDING REMARKS}

In summary, energy minimization relaxation occurs naturally when new surfaces are created with a free-standing film subjected no external loads. After relaxation, the film is at equilibrium and deformed with respect to its stress-free bulk counterpart. The relaxation-induced stress field is a must self-balanced internal stress field. In this paper, a fundamental energy-based continuum mechanics approach is proposed for the study of surface stress, surface energy, surface elastic constants of solids, and the surface-induced intrinsic thickness-dependent Young's modulus. To illustrate the physical picture of the developed eigenstress model, we take (001) films of crystals in the cubic crystal system, which simplifies the mathematical analysis greatly. The theoretical analysis and methodology developed in the present work are, however, universal when the continuum concepts of surface energy, surface stress, and surface elastic constants are used in academic research, in particular, in materials and mechanics research related to surfaces of solids. Moreover, the developed theoretical analysis and methodology should have the ability to investigate interfaces in solids.

\section{ACKNOWLEDGMENTS}

The work was supported by RGC Central Allocation Research Grants No. HKUST15/CRF/08 and No. PolyU7/ CRF/08 from the Hong Kong Research Grants Council, Hong Kong, China. Z.-J.W. was partially supported by the Nanotechnology Program, HKUST. 
*Corresponding author. FAX: (852) 2358-1543; mezhangt@ust.hk

${ }^{1}$ R. Shuttleworth, Proc. Phys. Soc., London, Sect. A 63, 444 (1950).

${ }^{2}$ M. M. Nicolson, Proc. Phys. Soc., London, Sect. A 228, 490 (1955).

${ }^{3}$ E. Orowan, Proc. Phys. Soc., London, Sect. A 316, 473 (1970).

${ }^{4}$ R. C. Cammarata, Prog. Surf. Sci. 46, 1 (1994).

${ }^{5}$ H. Ibach, Surf. Sci. Rep. 29, 195 (1997).

${ }^{6}$ R. E. Miller and V. B. Shenoy, Nanotechnology 11, 139 (2000).

${ }^{7}$ V. B. Shenoy, Phys. Rev. B 71, 094104 (2005).

${ }^{8}$ P. Müller and A. Saúl, Surf. Sci. Rep. 54, 157 (2004).

${ }^{9} \mathrm{H}$. Ibach, Physics of Surfaces and Interfaces (Springer-Verlag, Berlin, 2006).

${ }^{10}$ J. Weissmüller and D. Kramer, Langmuir 21, 4592 (2005).

${ }^{11}$ M. E. Gurtin and A. I. Murdoch, Arch. Ration. Mech. Anal. 57, 291 (1975).

${ }^{12}$ M. E. Gurtin and A. I. Murdoch, Int. J. Solids Struct. 14, 431 (1978).

${ }^{13}$ A. I. Murdoch, Q. J. Mech. Appl. Math. 29, 245 (1976).

${ }^{14}$ M. E. Gurtin, J. Weissmuller, and F. Larche, Philos. Mag. A 78, 1093 (1998).

${ }^{15}$ T. Y. Chen, M. S. Chiu, and C. N. Weng, J. Appl. Phys. 100, 074308 (2006).

${ }^{16}$ R. Dingreville, J. Qu, and M. Cherkaoui, J. Mech. Phys. Solids 53, 1827 (2005).

${ }^{17}$ R. Dingreville and J. Qu, Acta Mater. 55, 141 (2007).

${ }^{18}$ J. K. Diao, K. Gall, M. L. Dunn, and J. A. Zimmerman, Acta Mater. 54, 643 (2006).
${ }^{19}$ H. Liang, M. Upmanyu, and H. Huang, Phys. Rev. B 71, 241403 (2005).

${ }^{20}$ W. J. Huang, R. Sun, J. Tao, L. D. Menard, R. G. Nuzzo, and J. M. Zuo, Nat. Mater. 7, 308 (2008).

${ }^{21}$ J. K. Diao, K. Gall, and M. L. Dunn, J. Mech. Phys. Solids 52, 1935 (2004).

${ }^{22}$ T. Y. Zhang, M. Luo, and W. K. Chan, J. Appl. Phys. 103, 104308 (2008)

${ }^{23}$ J. W. Gibbs, The Collected Works of J. Willard Gibbs (Longmans, New York, 1928).

${ }^{24}$ H. L. Duan, J. Wang, Z. P. Huang, and B. L. Karihaloo, J. Mech. Phys. Solids 53, 1574 (2005).

${ }^{25}$ S. Plimpton, J. Comput. Phys. 117, 1 (1995).

${ }^{26}$ M. I. Baskes, Phys. Rev. B 46, 2727 (1992).

${ }^{27}$ M. I. Baskes and R. A. Johnson, Modell. Simul. Mater. Sci. Eng. 2, 147 (1994).

${ }^{28}$ M. S. Daw and M. I. Baskes, Phys. Rev. B 29, 6443 (1984).

${ }^{29}$ S. M. Foiles, M. I. Baskes, and M. S. Daw, Phys. Rev. B 33, 7983 (1986).

${ }^{30}$ J. Tersoff, Phys. Rev. B 38, 9902 (1988).

${ }^{31}$ P. Nozières and D. E. Wolf, Z. Phys. B: Condens. Matter 70, 399 (1988).

${ }^{32}$ S. Izumi, S. Hara, I. Kumagai, and S. Sakai, Thin Solid Films 467, 253 (2004).

${ }^{33}$ W. K. Chan, M. Luo, and T. Y. Zhang, Scr. Mater. 59, 692 (2008).

${ }^{34}$ W. K. Chan and T. Y. Zhang, J. Appl. Phys. 107, 023526 (2010). 